\title{
Downregulation of eIF4G by microRNA-503 enhances drug sensitivity of MCF-7/ADR cells through suppressing the expression of $\mathrm{ABC}$ transport proteins
}

\author{
XIA PAN, XIAOYAN YANG, JINGLEI ZANG, SI ZHANG, NAN HUANG, \\ XINXIN GUAN, JIANHUA ZHANG, ZHIHUI WANG, XI LI and XIAOYONG LEI \\ Institute of Pharmacy and Pharmacology, University of South China, Hengyang, Hunan 421001, P.R. China
}

Received March 4, 2016; Accepted February 23, 2017

DOI: $10.3892 / \mathrm{ol} .2017 .6049$

\begin{abstract}
Overexpression of adenosine triphosphate-binding cassette $(\mathrm{ABC})$ transport protein is emerging as a critical contributor to anticancer drug resistance. The eukaryotic translation initiation factor (eIF) $4 \mathrm{~F}$ complex, the key modulator of mRNA translation, is regulated by the phosphoinositide 3-kinase-AKT-mammalian target of rapamycin pathway in anticancer drug-resistant tumors. The present study demonstrated the roles of $\mathrm{ABC}$ translation protein alterations in the acquisition of the Adriamycin (ADM)-resistant phenotype of MCF-7 human breast cells. Quantitative polymerase chain reaction and western blot analysis were applied to examine the differences in mRNA and protein levels, respectively. It was found that the expression of the ABC sub-family B member 1, $\mathrm{ABC}$ sub-family $\mathrm{C}$ member 1 and $\mathrm{ABC}$ sub-family $\mathrm{G}$ member 2 transport proteins were upregulated in MCF-7/ADR cells. An MTT assay was used to detect the cell viability, from the results MCF-7/ADR cells were less sensitive to ADM, tamoxifen (TAM) and taxol (TAX) treatment compared with MCF-7 cells. We predicted that the 3'-untranslated region of eukaryotic translation initiation factor $4-\gamma 1$ (eIF4G) contains a potential miRNA binding site for microRNA (miR)-503 through using computational programs. These binding sites were confirmed by luciferase reporter assays. eIF4G mRNA degradation was accelerated in cells transfected with miR-503 mimics. Furthermore, it was demonstrated that eIF4G and $\mathrm{ABC}$ translation proteins were significantly downregulated in MCF-7/ADR cells after transfection with miR-503. It was found that miR-503 mimics could sensitize the cells to treatment with ADM, TAM and TAX. These findings demonstrated for the first time that eIF4G acted as a key factor in MCF-7/
\end{abstract}

Correspondence to: Professor Xiaoyong Lei, Institute of Pharmacy and Pharmacology, University of South China, 28 Changsheng Road, Hengyang, Hunan 421001, P.R. China

E-mail: 1622214323@qq.com

Key words: MCF-7/ADR, multidrug resistance, microRNA, eukaryotic translation initiation factor $4-\gamma 1$, breast cancer
ADR cells, and may be an efficient agent for preventing and reversing multi-drug resistance in breast cancer.

\section{Introduction}

Breast cancer is the most common disease in women worldwide. Cancer cells that have developed resistance to chemotherapeutic agents are major clinical obstacles in the successful treatment of breast cancer (1). When treated with one chemotherapeutic agent, patients can develop either intrinsical or acquired resistance to other drugs during the course of treatment (2). Chemotherapy drug resistance involves various mechanisms, including overexpression of adenosine triphosphate-binding cassette (ABC) transport proteins, changes in the expression of glutathione, glutathione transferase and topoisomerase, the enhancement of cancer cell DNA damage repair mechanisms and programmed cell death pathway defects (3-6). At present, acquired drug resistance and metastasis prevent breast cancer from being successfully treated in every case (7). It is necessary to improve the clinical management of cancer by increasing the sensitivity of tumor cells to chemotherapeutic agents and via the prediction of the effectiveness of chemotherapeutic agents, all without drug resistance developing in individual patients.

ABC transporters contribute to the development of the resistance of cancer cells to anticancer drugs via ATP-dependent drug efflux (1). Drug efflux in tumor cells is most frequently associated with overexpression of one or more membrane-bound ABC transporters (3). ABC sub-family B member 1 (ABCB1/P-gp) overexpression has been associated with poor drug response in breast cancer patients (8). ATP-binding cassette sub-family $\mathrm{C}$ member 1 (MRP1/ $\mathrm{ABCC} 1$ ) is involved in the multidrug resistance (MDR) of a variety of solid tumors (9). ABC sub-family $\mathrm{G}$ member 2 (ABCG2) encoding BCRP also belongs to the frequently studied ABC transporters (10). However, the role of other $\mathrm{ABC}$ genes in MDR is reported far less. Regulation of drug efflux is a key mechanism involeved in drug resistance. The results of the study by Pogribny et al (11) determinded that cisplatin-resistance cells upregulated MRP1 when compared with sensitive MCF-7 cells. 
The eukaryotic initiation factor (eIF) 4F complex consists of three proteins: cap-binding protein eIF4E, scaffolding protein eIF4G and ATP-dependent RNA helicase eIF4A $(12,13)$. All three proteins converge to modulate the translation of specific mRNAs. Generally, 4EBP1 inhibits the downstream mTOR pathway through binding to eIF4E. Phosphorylation of 4EBP1 by mTOR results in its dissociation from eIF4E and activation of cap-dependent mRNA translation (14). The increased amount of 4EBP1-bound eIF4E concomitantly decreases the amount of eIF4G-bound eIF4E and vice versa. The correct functioning of cellular processes, including drug resistance, is regulated by controling gene expression at the mRNA translational level $(15,16)$. Recently, eIF4F complex formation was found to be reduced in tumors responsive to anti-BRAF therapy, but increased in resistant metastases, compared with tumors prior to treatment. B-cell lymphoma-2 modifying factor (BMF) is a pro-apoptotic gene that has previously been demonstrated to be involved in the acquired resistance to PLX4720, a vemurafenib analogue. BMF has also demonstrated involvment in the sensitivity to vemurafenib by acting on the cleavage of eIF4G, which consequently affects the formation of the eIF4F complex (17). This phenomenon is similar to the drug resistance of breast cancer cells caused by high expression of the ABC transporter family. Although drug resistance can be reversed by disrupting the eIF4F complex, the association between eIF4F and $\mathrm{ABC}$ transporters is not clear.

The present study investigated the potential role of eIF4G in the resistance of MCF-7/ADR cells to anticancer drugs, and unravelled the possible association between eIF4G and ABC transporters.

\section{Materials and methods}

Cells and reagents. The human breast cancer MCF-7 cell line, maintained at $37^{\circ} \mathrm{C}$ with $5 \% \mathrm{CO}_{2}$ in a humidified atmosphere and grown in Dulbecco's modified Eagle's medium (HyClone Laboratories; GE Healthcare, Logan, UT, USA) supplemented with $10 \%$ fetal bovine serum (FBS; Gibco; Thermo Fisher Scientific, Inc., Waltham, MA, USA), was a gift from the Institute of Pharmacy and Pharmacology, University of South China (Hengyang, Hunan, China). The MCF-7 Adriamycin (ADM)-resistant (MCF-7/ADR) cell line was alternately fed with medium containing $1 \mu \mathrm{g} / \mathrm{ml}^{-1}$ ADM (Sigma-Aldrich; Merck KGaA, Darmstadt, Germany) and RPMI-1640 medium supplemented with $10 \%$ FBS, and was regularly tested for maintenance of drug-resistaince. The cells were treated with ADM (Sigma-Aldrich; Merck KGaA), tamoxifen (TAM; Sigma-Aldrich; Merck KGaA) and taxol (TAX; Sigma-Aldrich; Merck KGaA). All drugs were dissolved in dimethyl sulfoxide (DMSO) for in vitro studies.

Target prediction. Several online Bioinformatics and Research software programs, TargetScan (http://www.targetscan. org), miRanda (http://www.microrna.org/), Pictar (https:// www.mdc-berlin.de/10440258/en/research/researchteams/ systemsbiology ofgeneregulatoryelements/projects/pictar) and miRBase (http://www.mirbase.org/search.shtml) were used to predict conserved miRNA binding sites in the 3'-untranslated region (3'UTR) of human eIF4G.
Transient transfection. miR-503 mimic, miRNA mimic control and miR-503 inhibitor (all GenePharma, Shanghai, China) marked with carboxyfluorescein (FAM) in vitro were transfected into the cells at a final concentration of $160 \mathrm{nM}$ using Lipofectamine 2,000 (Invitrogen Life Technologies; Thermo Fisher Scientific, Inc.) according to the manufacturer's protocols. After $6 \mathrm{~h}$, fluorescence microscopy was used to detect the percent of fluorescent cells. After $48 \mathrm{~h}$, western blot analysis was performed.

Western blot analysis. Western blot analysis was performed on cell extracts of the indicated cell lines that had been transfected for $48 \mathrm{~h}$ with the miRNA sequences. Immunoblots were performed from whole cell lysate prepared using RIPA Buffer supplemented with PMSF, and phosphatase inhibitors. Cell lysates were quantified for protein content using a bicinchoninic acid protein assay kit. SDS PAGE was used to separate the proteins, with $30 \mu \mathrm{g}$ total protein per lane on 5\% SDS-PAGE. Protein samples were transferred to $0.22-\mathrm{mm}$ polyvinyl difluoride membranes. After saturation in Tris-buffered saline with Tween-20 and 5\% powdered milk, the membranes were incubated with anti-eIF4G (cat. no., $2617 \mathrm{~S}$; dilution, 1:1,000) and anti- $\beta$-actin (cat. no., $8457 \mathrm{~S}$; dilution, 1:1,000) antibodies purchased from Cell Signaling Technology, ABCB1 (cat. no., ab170904; dilution, 1:1,000), ABCC1 (cat. no., ab3368; dilution, 1:1,000) and ABCG2 (cat. no., ab108312; dilution, 1:1,000) purchased from Abcam, overnight at $4^{\circ} \mathrm{C}$. The membranes were then incubated with horseradish peroxidase-conjugated secondary antibodies (cat. no., sc-2357; dilution, 1:5,000 Santa Cruz Biotechnology, Inc., Dallas, TX, USA) for $1 \mathrm{~h}$ at room temperature. The signals were visualized using ECL Substrates (Tanon Ltd, shanghai, China) and quantified using AlphaImager 2200 software.

Reverse transcription-quantitative polymerase chain reaction (RT-qPCR). Total RNA was extracted from cells using the TRIzol (Invitrogen; Thermo Fisher Scientific, Inc.) chloroform extraction method. The cDNA was prepared from $1 \mu \mathrm{g}$ total RNA using reverse transcriptase (Promega Corporation, Madison, WI, USA). Total cellular RNA was quantified by qPCR analysis using gene-specific primer pairs. RT-qPCR was performed by the SYBR-Green qPCR Master Mix (Tiangen Biotech Co., Ltd., Beijing, China) on an ABI 7300 PCR machine (Applied Biosystems, Inc., Foster, CA, USA). qPCR cycling conditions were as follows: $95^{\circ} \mathrm{C}$ for $5 \mathrm{~min}$, and then $95^{\circ} \mathrm{C}$ for $15 \mathrm{sec}, 65^{\circ} \mathrm{C}$ for $15 \mathrm{sec}$ and $72^{\circ} \mathrm{C}$ for $32 \mathrm{sec}$, for 40 cycles. The melting curve was $60-95^{\circ} \mathrm{C}$. The relative mRNA expression levels were calculated as $2^{-\Delta \Delta C q}$ method (18). Oligonucleotides used for the analysis were as follows: ABCB1 forward, 5'-CTAGAAGGTTCTGGGAAG AT and reverse, 5'-GAGTTTCTGTATGGTACCTG; ABCC1 forward, 5'-GGCTCAAGGAGTATTCAGAG and reverse, 5'-CCATCGATGATGATCTCTCC; EIF4G forward, 5'-CTC TGAGACCGTTGGGCAAA and reverse, 5'-GCGACGAAT GCACCAATGT; ABCG2 forward, 5'-GCAGGTCAGAGT GTGGTTTC and reverse, 5'-GACAGCCAAGATGCAATG GT; and 18S ribosomal RNA forward, 5'-CCTGGATACCGC AGCTAGGA and reverse, 5'-GCGGCGCAATACGAATGC CCC. Each reaction was performed in triplicate and three independent experiments were run. 
A
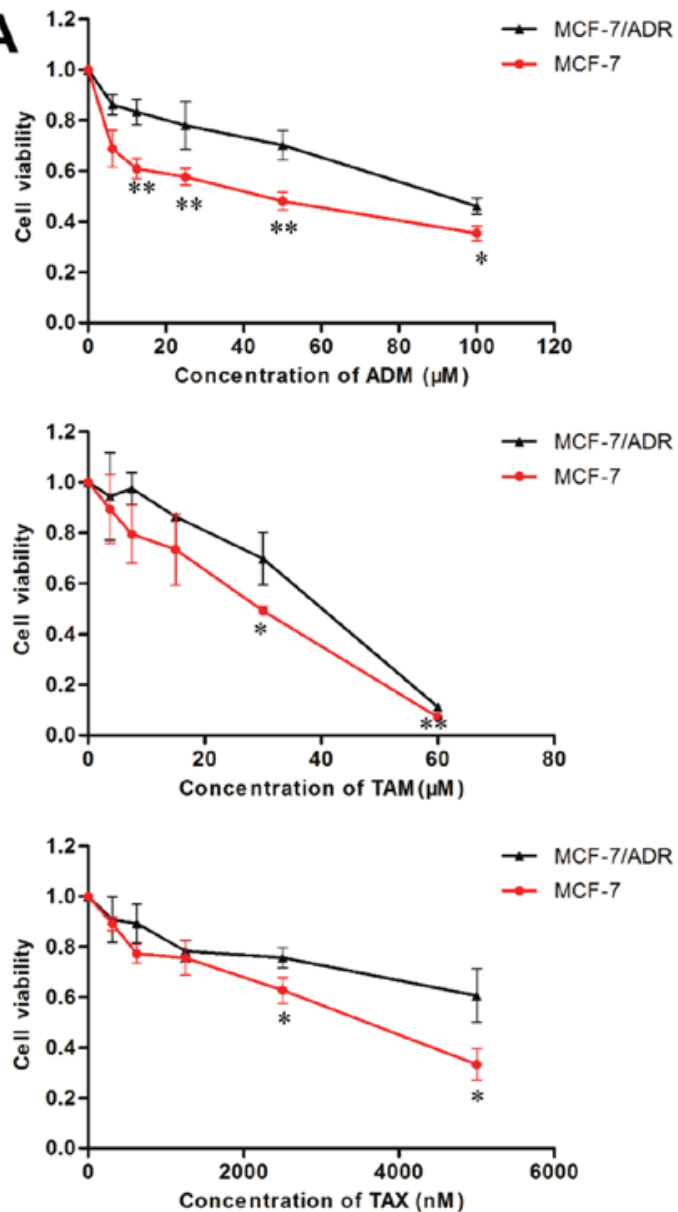

B

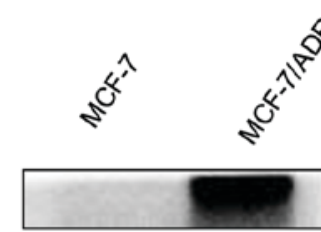

ABCB1

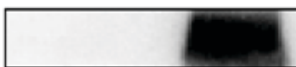

$\mathrm{ABCC} 1$

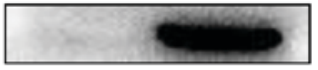

ABCG2

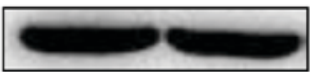

$\beta$-actin

C

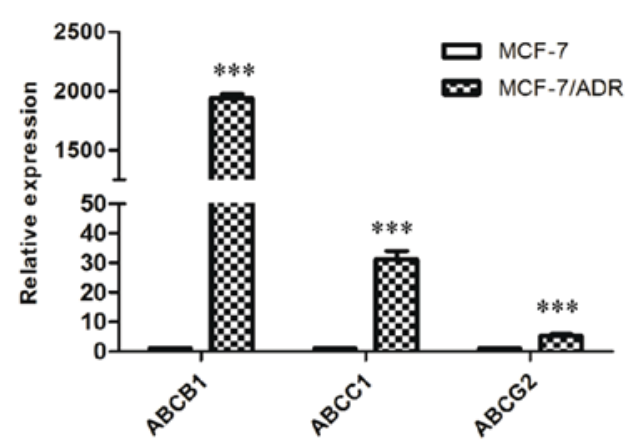

Figure 1. Upregulation of ABCB1, ABCC1 and ABCG2, and alterations in ADM, TAM and TAX sensitivity in MCF-7/ADR cells. (A) MTT analyses showed that MCF-7 cells were sensitive to ADM, TAM and TAX compared with the MCF-7/ADR cells ( $* \mathrm{P}<0.05, * * \mathrm{P}<0.01$ ). (B) Western blotting analysis of ABCB1, $\mathrm{ABCC} 1$ and $\mathrm{ABCG} 2$ in the indicated cell lines. $\beta$-actin was used as a loading control. (C) Expression levels of ABCB1, ABCC1 and ABCG2 mRNA in the MCF-7/ADR cells, as determined by reverse transcription-quantitative polymerase chain reaction, were upregulated in comparison with the MCF-7 cells (***P<0.001). ABCB1, ABC sub-family B member 1; ABCC1, ABC sub-family C member 1; ABCG2, ABC sub-family G member 2; ADM, Adriamycin; TAM, tamoxifen; TAX, taxol.

\section{Position 340-346 of elF4G1 3'UTR 5' ...CGGGACUGCCGUCCUCGCUGCUG... \\ hsa-miR-503 \\ 3' GACGUCUUGACAAGG - - -GCGACGAU}

Figure 2. Putative miR-503 targeted sequence in the eIF4G gene. miR, microRNA; eIF4G, eukaryotic translation initiation factor 4- $\gamma$ 1; UTR, untranslated region.

$M T T$. Cells in the exponential growth phase were seeded in 96-well plates at a density of $1 \times 10^{4}$ per well. After incubation for $24 \mathrm{~h}$ at $37^{\circ} \mathrm{C}$ with $5 \% \mathrm{CO}_{2}$ in a humidified atmosphere, the cells were transfected with miR-503 mimic, miR-503 inhibitor or miRNA mimic control (NC). At $24 \mathrm{~h}$ post-transfection, various concentrations of $\operatorname{ADM}(0,0.625,12.5,25$, 50 and $100 \mu \mathrm{M})$, TAX $(0,0.3125,0.625,1.25,2.5$ and $5 \mu \mathrm{M})$ and TAM $(0,3.75,7.5,15,30$ and $60 \mu \mathrm{M})$ were added. After an incubation period of $24 \mathrm{~h}, 20 \mu \mathrm{l}$ MTT solutions with a concentration of $5 \mathrm{mg} / \mathrm{ml}$ were added to each well for $4 \mathrm{~h}$ at $37^{\circ} \mathrm{C}$. Next, the culture medium (medium containing drug and MTT) was removed, and the insoluble formazan crystals were dissolved in $150 \mathrm{ml}$ DMSO. After shaking for $10 \mathrm{~min}$, the absorbance at $570 \mathrm{~nm}$ was optically monitored using a microplate reader.
Luciferase reporter assay. The wild-type or mutant eIF4G 3'UTR-luciferase reporter plasmids (Landbiology, Guangzhou, China) were co-transfected with miR-503 mimics, miR-503 control (NC), miR-503 inhibitor and inhibitor control (NC inhibitor) into the MCF-7 and MCF-7/ADR cells. At $48 \mathrm{~h}$ post-transfection, the cells were assayed for luciferase activity using the Dual Luciferase assay system (Promega Corporation) according to the manufacturer's instructions. The Renilla luciferase activities were normalized to corresponding firefly luciferase activities. For each transfection, the luciferase activity was averaged from three replicates.

Assessment of cell cycle and apoptosis. At $48 \mathrm{~h}$ post-transfection, the cells were washed with phosphate-buffered saline and fixed with $70 \%$ ethanol overnight. The cells were then 

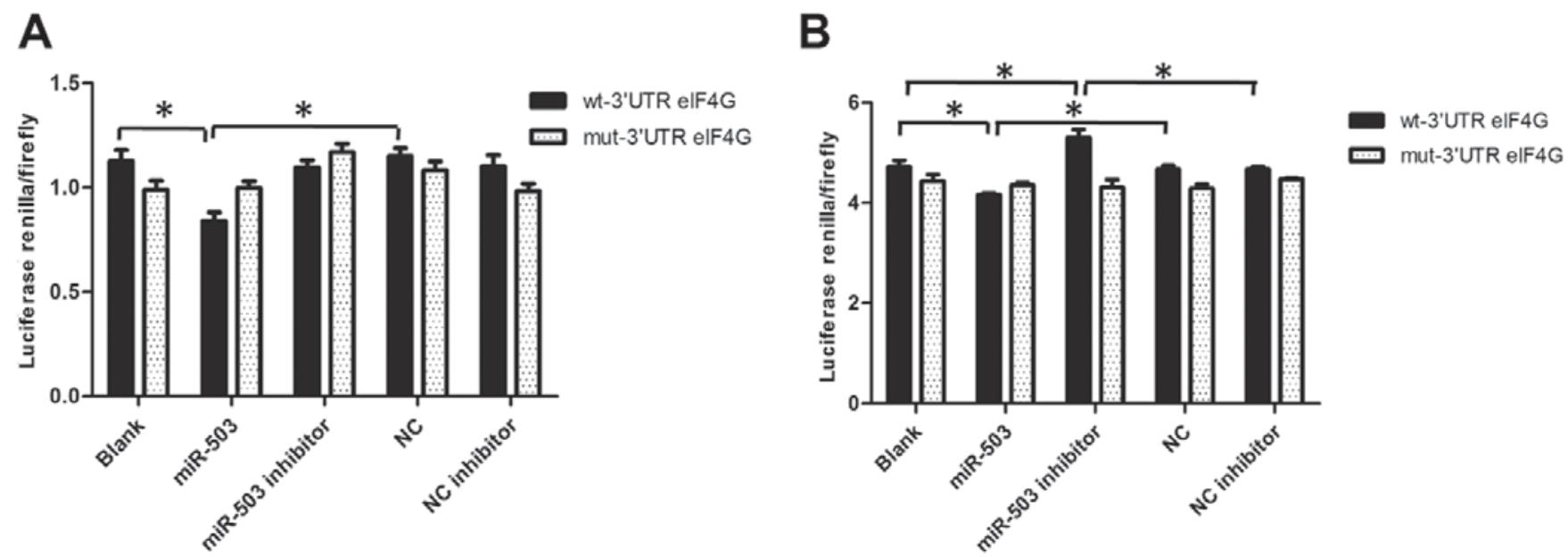

Figure 3. miR-503 target identification in MCF-7 and MCF-7/ADR cells. Luciferase reporter assays confirmed that eIF4G was a direct target of miR-503 in (A) MCF-7 and (B) MCF-7/ADR cells (*P<0.05). miR, microRNA; eIF4G, eukaryotic translation initiation factor 4- $\gamma$ 1; UTR, untranslated region; NC, negative control.

\section{A}
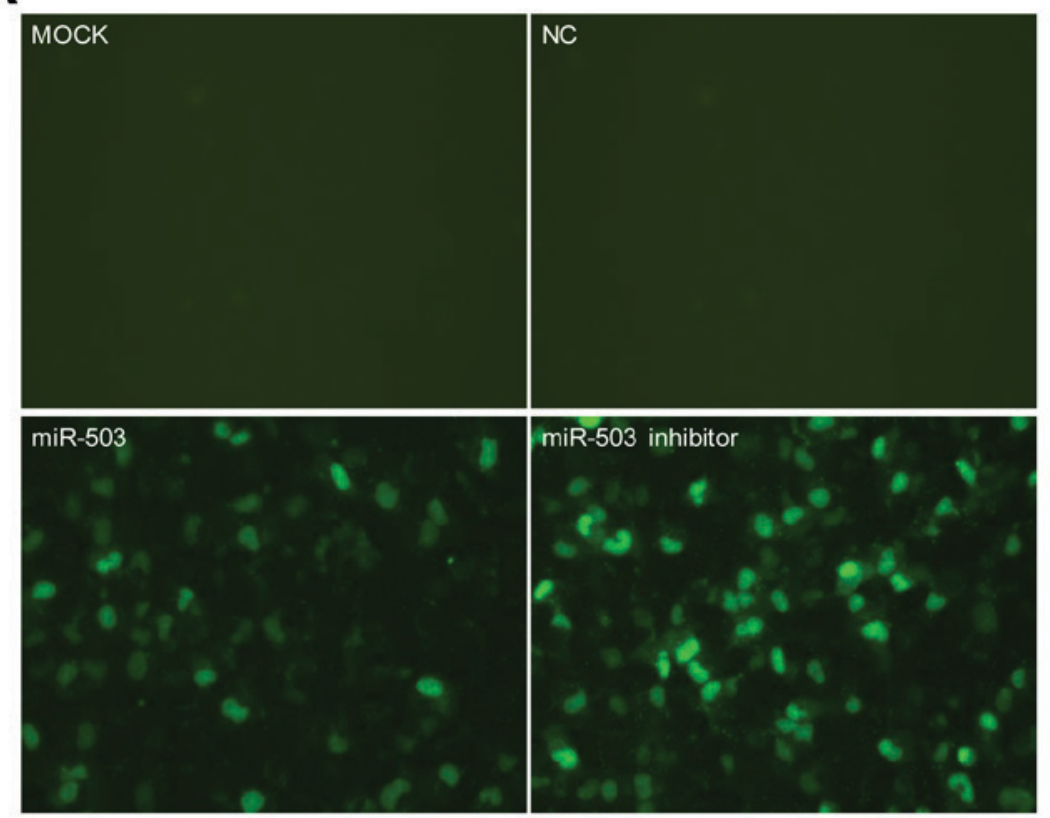

\section{D}

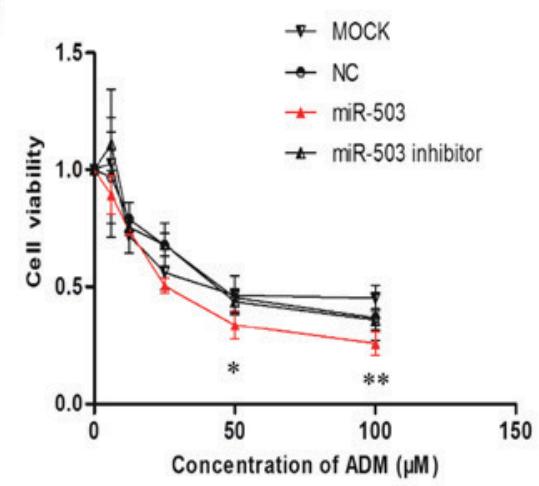

B
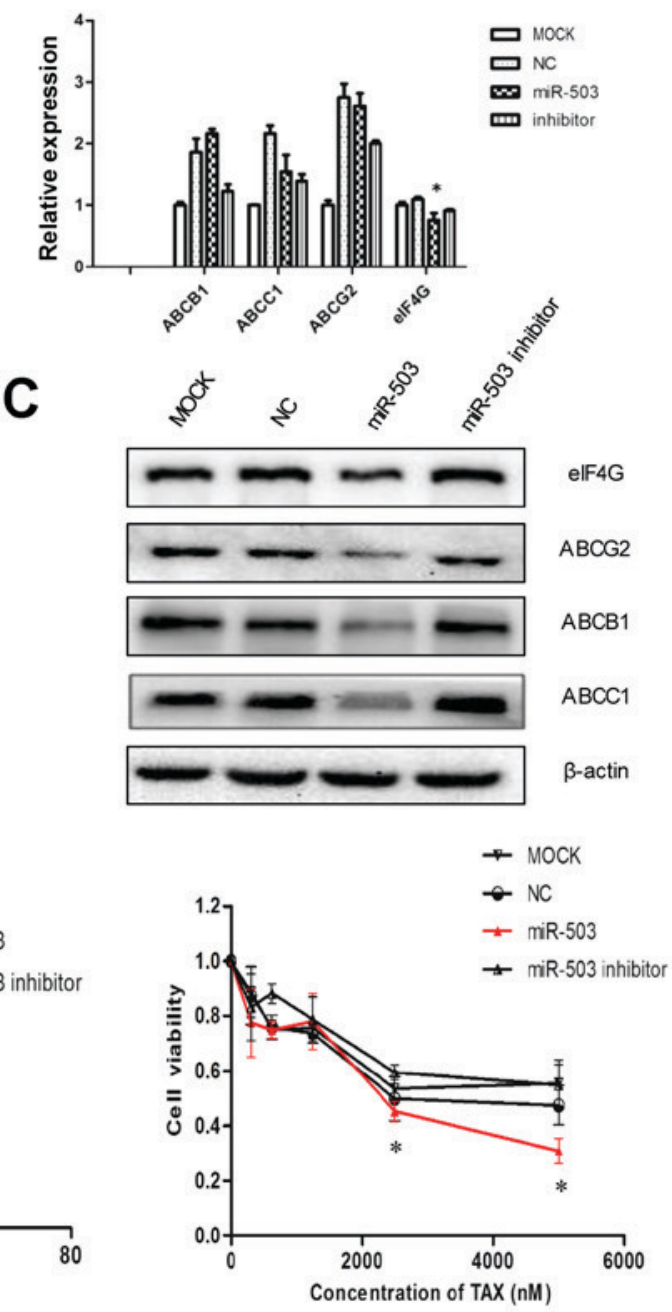

Figure 4. miR-503 negatively regulates the expression of ABCB1, ABCC1 and ABCG2, and increases MCF-7/ADR sensitivity to ADM, TAX and TAM. (A) After $48 \mathrm{~h}$, fluorescence microscopy was used to detect transfection efficiency, and the transfection efficiency of the miR-503 and miR-503 inhibitor was $>35 \%$. (B) The expression of eIF4G mRNA was downregulated, while the expression levels of ABCB1, ABCC1 and ABCG2 mRNA in the miR-503 mimic-transfected MCF-7/ADR cells were not changed in comparison with the control cells and untreated cells, as determined by reverse transcription-quantitative polymerase chain reaction ( ${ }^{* *} \mathrm{P}<0.01$ vs. negative microRNA or mock group). (C) miR-503 negatively regulated the expression of eIF4G, $\mathrm{ABCB} 1, \mathrm{ABCC} 1$ and ABCG2, as determined by western blot assay. (D) miR-503 inhibited drug resistance, as determined by MTT assay $\left({ }^{*} \mathrm{P}<0.05,{ }^{* *} \mathrm{P}<0.01\right)$. 

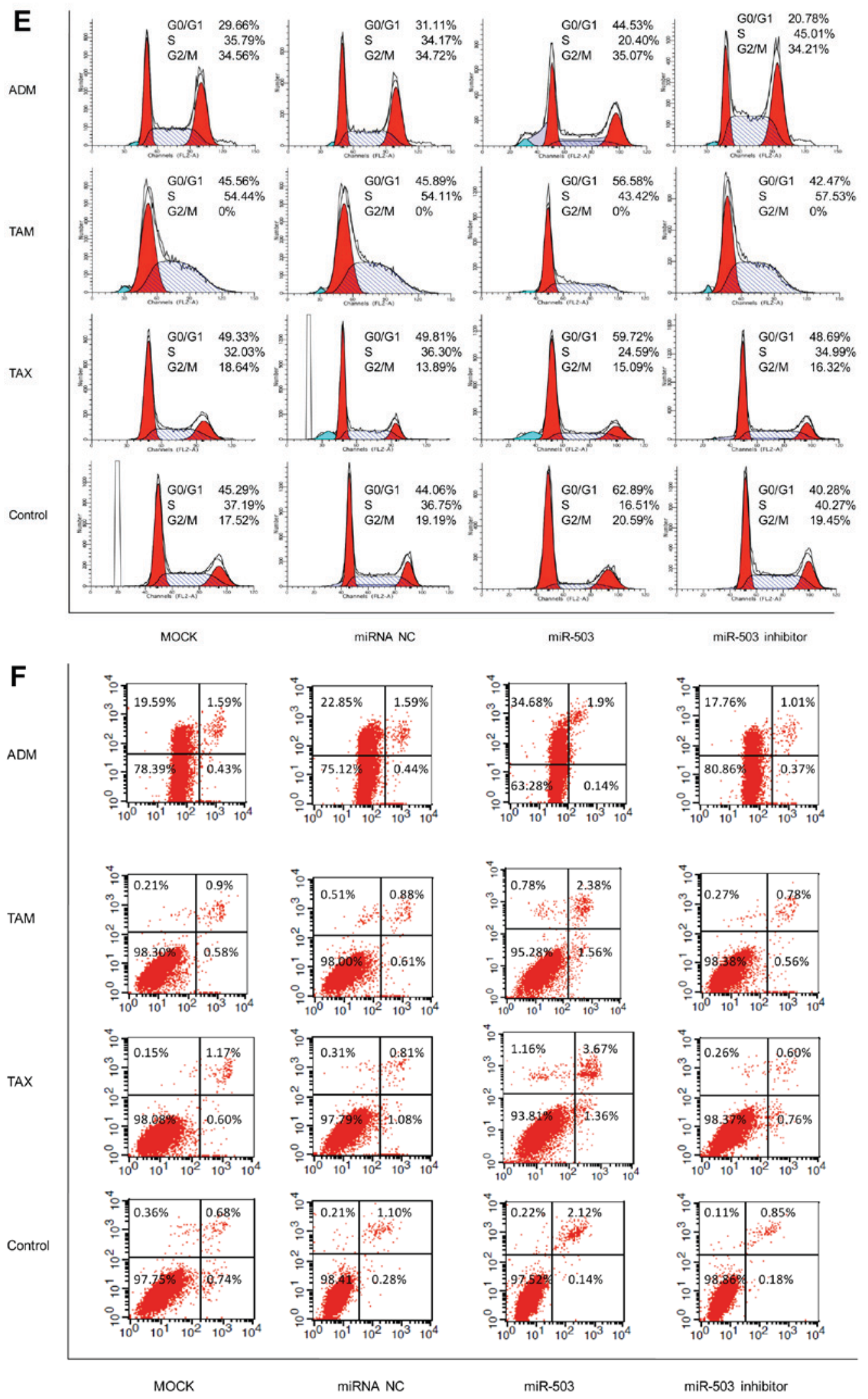

Figure 4. Continued. (E) Cell cycle distributions were analyzed with flow cytometry, MCF-7/ADR cells transfected with miR-503 were arrested in G0/G1 phases. (F) miR-503 increased the percentage of apoptosis cells treated with anticancer drugs ( $20 \mu \mathrm{M} \mathrm{ADM}, 10 \mu \mathrm{M}$ TAM and $5 \mu \mathrm{M}$ TAX). ABCB1, ABC sub-family B member 1; ABCC1, ABC sub-family C member 1; ABCG2, ABC sub-family G member 2; ADM, Adriamycin; TAM, tamoxifen; TAX, taxol; miR, microRNA; eIF4G, eukaryotic translation initiation factor 4- $\gamma 1$; NC, negative control.

stained with $50 \mu \mathrm{g} / \mathrm{ml}$ propidium iodide, $100 \mu \mathrm{g} / \mathrm{ml}$ RNase $\mathrm{A}$ and $0.2 \%$ Triton X-100 (Nanjing KeyGen Biotech, Co.,
Ltd.; KGA511), and kept out of the light for 30 min, prior to undergoing cell cycle analysis using a flow cytometer. The 

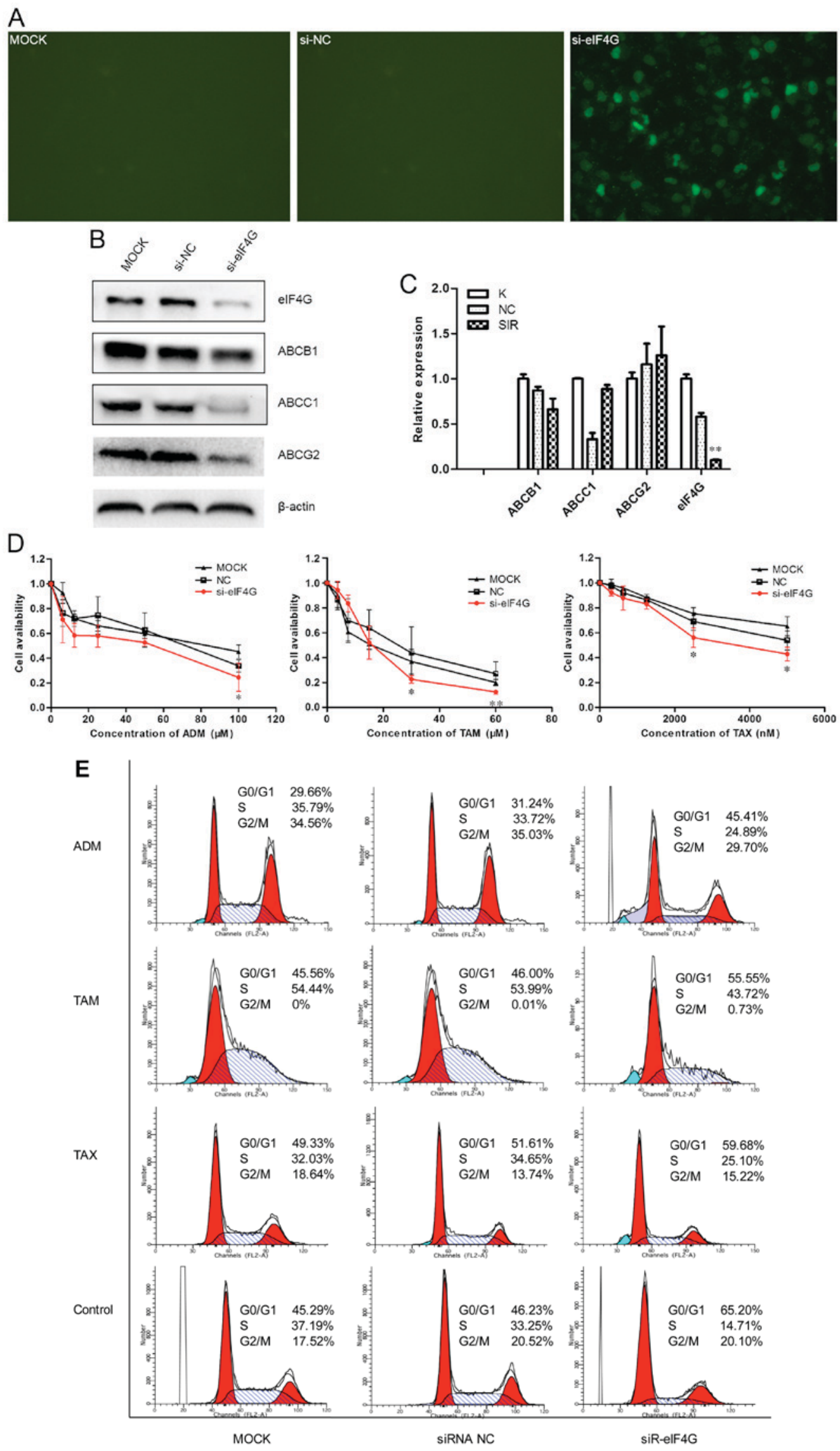

Figure 5. eIF4G siRNA negatively regulated the expression of ABCB1, ABCC1 and ABCG2, and increased MCF-7/ADR sensitivity to ADM, TAX and TAM. (A) Transfection efficiency of eIF4G siRNA and NC siRNA. After $48 \mathrm{~h}$, fluorescence microscopy was used to detect transfection efficiency, and the transfection efficiency was $>35 \%$. (B) eIF4G siRNA negatively regulated the expression of ABCB1, ABCC1 and ABCG2, as assessed by western blot assay. (C) The effect of eIF4G siRNA on mRNA. (D) eIF4G siRNA reduced cell viability compared with siRNA NC or untransfected cultures, as determined by MTT assay $\left({ }^{*} \mathrm{P}<0.05,{ }^{* *} \mathrm{P}<0.01\right)$. (E) Cell cycle distributions were analyzed by flow cytometry, and MCF-7/ADR cells transfected with eIF4G siRNA were arrested in G0/G1 phases. 


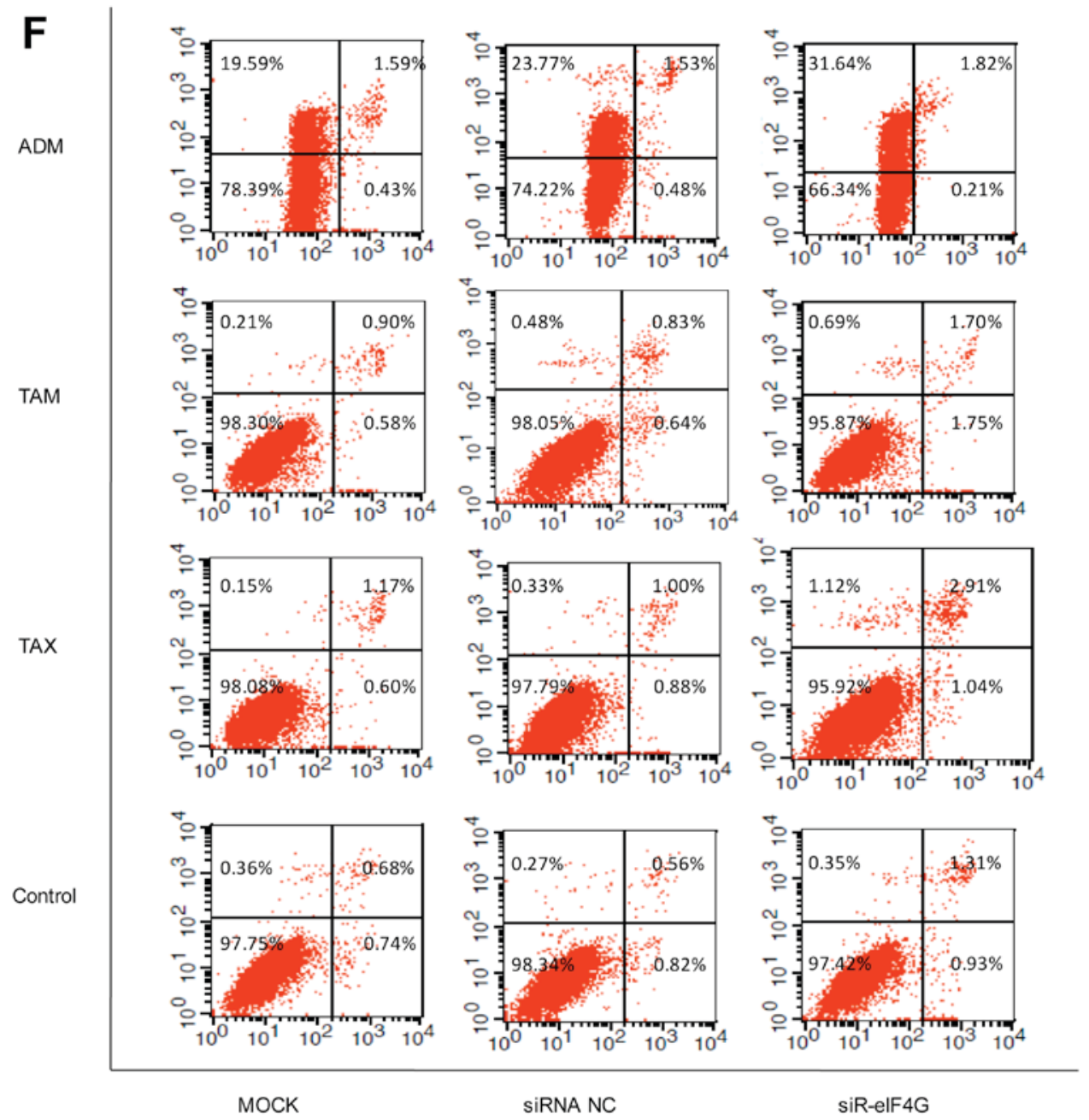

Figure 5. Continued. (F) eIF4G siRNA increased the percentage of apoptotic cells treated with anticancer drugs (20 $\mu \mathrm{M}$ ADM, $10 \mu \mathrm{M}$ TAM and $5 \mu \mathrm{M}$ TAX). eIF4G, eukaryotic translation initiation factor 4- $\gamma$ 1; siRNA, small interfering RNA; ABCB1, ABC sub-family B member 1; ABCC1, ABC sub-family C member 1; ABCG2, ABC sub-family G member 2; ADM, Adriamycin; TAM, tamoxifen; TAX, taxol; NC, negative control.

percentage of apoptotic cells was determined by flow cytometry (BD calibur, USA) with the Annexin V staining kit (cat. no., KGA106; Nanjing KeyGen Biotech, Co., Ltd.).

Statistical analysis. All experiments were performed at least three times $(n=3)$. Statistical analyses used the two-tailed Student's $t$-test, with $\mathrm{P}<0.05$ used to indicate a statistically significant difference. Statistical analysis was performed using GraphPad Prism 5.00 (GraphPad, San Diego, CA, USA).

\section{Results}

$A B C B 1, A B C C 1$ and $A B C G 2$ were overexpressed in $M C F-7 /$ $A D R$ cells. To analyze the alterations in ABCB1, ABCC1 and $\mathrm{ABCG} 2$ during the formation of breast cancer cellular drug resistance, the MCF-7/ADR cell line, an ADM-resistant variant, was utilized and confirmed to be more resistant compared with the MCF-7 cells treated with ADM, TAM and TAX (Fig. 1A). It was identified that the ATP-dependent efflux genes (ABCB1, ABCC1 and ABCG2) exhibited higher protein and mRNA levels in the MCF-7/ADR cells compared to the parental MCF-7 cells (Fig. 1B and C).
3'UTR of eIF4G gene contains conserved miRNA binding sites for miR-503. miR-503 was predicted to bind the 3'UTR of human eIF4G by bioinformatics and research computing programs, including TargetScan, Pictar and miRanda (Fig. 2).

Putative miRNAs binding sites for miR-503 were confirmed by luciferase reporter assays. To validate whether the 3 'UTR of human eIF4G could be recognized by miR-503, a luciferase reporter assay was utilized. The luciferase activity in the cells transfected with the vector containing the eIF4G 3'UTR fragment with the binding sequence of miR-503 was inhibited by miR-503 transfection compared with the activity of the cells that were transfected with the NC miRNA mimic, miR-503 inhibitor and the blank control (Fig. 3). From the results (Fig. 3A) in the MCF-7 group, it is apparent that the 3'UTR eIF4G wild-type inhibition rate was 0.75 and that the mut-3'UTR eIF4G inhibition rate was 1.01. Fig. 3B demonstrates that in MCF-7/ADR group, the 3'UTR eIF4G wild-type inhibition rate was 0.88 and the mut-3'UTR eIF4G inhibition rate was 0.98 , suggesting that the wild-type-3'UTR eIF4G serves a more important role in the binding association than the mut-3'UTR eIF4G. 
Elevated levels of miR-133 decreased eIF4G mRNA expression. The MCF-7/ADR cells were transfected with Lipofectamine 2000 (mock), miRNA control (NC), miR-503 and miR-503 inhibitor marked with FAM in vitro (Fig. 4A). After $48 \mathrm{~h}$, fluorescence microscopy was used to detect the transfection efficiency. The data (Fig. 4B) showed that miR-503 did not significantly affect the expression of ABCB1, ABCC1 or ABCG2 mRNA, but that it significantly downregulated the expression of eIF4G. These results demonstrated that ABCB1, $\mathrm{ABCC} 1$ and $\mathrm{ABCG} 2$ were not the target genes of miR-503, but that miR-503 could downregulate eIF4G mRNA expression.

miR-503 downregulates the expression of eIF4 G, ABCB1, $A B C C 1$ and $A B C G 2$ proteins, and increases MCF-7/ADR sensitivity to ADM, TAM and TAX. The present study assessed the function of miR-503 in human breast cancer, with a particular focus on MCF-7/ADR cells. It was revealed that miR-503 may cause the downregulation of eIF4G, ABCB1, $\mathrm{ABCC} 1$ and $\mathrm{ABCG} 2$ (Fig. 4C), and the sensitivity of the MCF-7/ADR cells to ADM, TAM and TAX was demonstrated to be increased by miR-503 (Fig. 4D). In addition, miR-503 was identified to be able to block the cell cycle at $\mathrm{G}_{0} / \mathrm{G}_{1}$ (Fig. 4E) and lead to the occurrence of apoptosis in MCF-7/ ADR cells (Fig. 4F). These results suggested that miR-503 may decrease the chemoresistance of breast cancer cells by the downregulation of eIF4G, ABCB1, ABCC1 and ABCG2.

eIF4G siRNA negatively regulates the expression of $A B C B 1$, $A B C C 1$ and $A B C G 2$, and increases MCF-7/ADR cell sensitivity to ADM, TAM and TAX. In transient cells transfected with eIF4G siRNA, after $6 \mathrm{~h}$ the transfection efficiency was $>35 \%$, as detected by fluorescence microscopy (Fig. 5A). Using RT-qPCR, the difference in eIF4G expression in the MCF-7/ADR cells transfected with siR-eIF4G was examined and was revealed to be significantly downregulated in comparison with that of the control cells and untreated cells (Fig. 5C). eIF4G siRNA negatively regulated the protein expression of $\mathrm{ABCB} 1, \mathrm{ABCC} 1$ and $\mathrm{ABCG} 2$, as determined by western blot assay (Fig. 5B), but exhibited no effect on the mRNA expression (Fig. 5C). The results (Fig. 5D) demonstrated that eIF4G siRNA reduced cell viability compared with siRNA NC or untransfected cultures, as assessed by MTT assay. In addition, eIF4G siRNA was identified to be able to block the cell cycle at $\mathrm{G}_{0} / \mathrm{G}_{1}$ (Fig. 5E) and lead to the occurrence of apoptosis in MCF-7/ADR cells (Fig. 5F).

\section{Discussion}

Several studies previously reported that $\mathrm{ABCB} 1, \mathrm{ABCC} 1$ and ABCG2 were upregulated in pre- and post-treatment breast tumors $(1,21)$. Despite notable progress in research, MDR remains the main obstacle to successful cancer treatment. ABC transporters are one of the major factors leading to chemoresistance owing to their ability to efflux drugs outside of tumor cells, thus diminishing the therapeutic effect (22). In the present study, an ADM-resistant breast cancer cell line was used as a model to address the role of $\mathrm{ABC}$ transport proteins in tumor cells. $\mathrm{ABCB} 1, \mathrm{ABCC} 1$ and $\mathrm{ABCG} 2$ were found to be overexpressed in the MCF-7/ADR cells, indicating that MCF-7/ADR tumor cells may develop MDR to a variety of chemotherapy drugs $(23,24)$. When treated with three types of drugs whose structures and mechanisms of action are completely different, the MCF-7/ADR cells were shown to be resistant to ADM, TAX and TAM when compared with the MCF-7 cells.

The molecular mechanism of eIF4G, a component of the translation initiation complex, awaits further clarification. Previous studies identified that eIF4G is a positive regulator of tumor growth via regulation of translation initiation activity. Accordingly, the corresponding inhibition of translation and induction of senescence by miRNAs and/or small molecule inhibitors targeting eIF4G are potential novel strategies for cancer treatment $(25,26)$. Increasing evidence shows that the abnormal function of the cellular eIF4F complex, which is caused by increased expression of initiation factors, serves a key role in tumorigenesis. It is a widely held belief that the targeting of the molecules of this complex may be used as a cancer therapy.

A number of studies have demonstrated that miRNAs are associated with numerous critical cellular pathways and that they are able to function together with other genes that are crucial for the control of cellular growth $(20,27)$. Accordingly, the results of our earlier studies provided an experimental basis for using these small molecules in a therapeutic approach (28), and miR-133a and miR-326 have been established as personalized therapy for HCC treatment. It is vital that the gene/pathway that these small regulatory molecules regulate is identified. We predicted that the 3'UTR of eIF4G contained one potential binding site for miR-503, and the present study confirmed the binding of miR-503 to the 3'UTR of eIF4G in breast cancer cells. Elevated levels of miR-503 were confirmed to lead to the inhibition of eIF4G gene translation and cell proliferation, and to promote senescence in MCF-7/ADR cells. A number of studies have demonstrated that disruption of the translational machinery one of the main contributors to the development and progression of cancer $(29,30)$. A large body of research has become focused upon investigating the mechanisms of translational regulation by miRNAs $(31,32)$. The present results provided important evidence of the regulation of translational initiation complex by the targeting of eIF4G by miRNA, and thereby the modulation of drug sensitivity in cancer cells.

In summary, the present study demonstated that miR-503 could increase the sensitivity of human MCF-7/ADR cells to ADM, TAX and TAM via targeting eIF4G, leading to the downregulation of $\mathrm{ABCB} 1, \mathrm{ABCC} 1$ and $\mathrm{ABCG} 2$ transport proteins. Targeting miR-503 would be a potential therapeutic strategy for the treatment of breast cancer with MDR.

\section{Acknowledgements}

This study was supported by the grants from the National Natural Science Foundation of China (no. 81372579), the Hunan Provincial Innovation Foundation For Postgraduate (no. 2015SCX29) and the Hunan Province Cooperative Innovation Center for Molecular Target New Drug Study.

\section{References}

1. Hlaváč V, Brynychová V, Václavíková R, Ehrlichová M, Vrána D, Pecha V, Koževnikovová R, Trnková M, Gatěk J, Kopperová D, etal: The expression profile of ATP-binding cassette transporter genes in breast carcinoma. Pharmacogenomics 14: 515-529, 2013. 
2. Gottesman MM, Hrycyna CA, Schoenlein PV, Germann UA and Pastan I: Genetic analysis of the multidrug transporter. Annu Rev Genet 29: 607-649, 1995

3. Lo YL and Liu Y: Reversing multidrug resistance in Caco-2 by silencing MDR1, MRP1, MRP2, and BCL-2/BCL-xL using liposomal antisense oligonucleotides. PLoS One 9: e90180, 2014.

4. Deeley RG, Westlake C and Cole SP: Transmembrane transport of endo- and xenobiotics by mammalian ATP-binding cassette multidrug resistance proteins. Physiol Rev 86: 849-899, 2006.

5. Gottesman MM, Fojo T and Bates SE: Multidrug resistance in cancer: Role of ATP-dependent transporters. Nat Rev Cancer 2: 48-58, 2002.

6. Krishnamurthy P and Schuetz JD: Role of ABCG2/BCRP in biology and medicine. Annu Rev Pharmacol Toxicol 46: 381-410, 2006.

7. Gong MQ, Wu JL, Chen B, Zhuo RX and Cheng SX: Self-assembled polymer/inorganic hybrid nanovesicles for multiple drug delivery to overcome drug resistance in cancer chemotherapy. Langmuir 31: 5115-5122, 2015.

8. Bao L, Hazari S, Mehra S, Kaushal D, Moroz K and Dash S: Increased expression of P-glycoprotein and doxorubicin chemoresistance of metastatic breast cancer is regulated by miR-298 Am J Pathol 180: 2490-2503, 2012.

9. Berger W, Hauptmann E, Elbling L, Vetterlein M, Kokoschka EM and Micksche M: Possible role of the multidrug resistanceassociated protein (MRP) in chemoresistance of human melanoma cells. Int J Cancer 71: 108-115, 1997.

10. Padmanabhan R, Chen KG, Gillet JP, Handley M, Mallon BS, Hamilton RS, Park K, Varma S, Mehaffey MG, Robey PG, et al Regulation and expression of the ATP-binding cassette transporter ABCG2 in human embryonic stem cells. Stem Cells 30: 2175-2187, 2012

11. Pogribny IP, Filkowski JN, Tryndyak VP, Golubov A, Shpyleva SI and Kovalchuk O: Alterations of microRNAs and their targets are associated with acquired resistance of MCF-7 breast cancer cells to cisplatin. Int J Cancer 127: 1785-1794, 2010.

12. Silvera D, Formenti SC and Schneider RJ: Translational control in cancer. Nat Rev Cancer 10: 254-266, 2010.

13. Li BD, McDonald JC, Nassar R and De Benedetti A: Clinical outcome in stage I to III breast carcinoma and eIF4E overexpression. Ann Surg 227: 756-761; 761-763, 1998.

14. Demosthenous C, Han JJ, Stenson MJ, Maurer MJ, Wellik LE, Link B, Hege K, Dogan A, Sotomayor E, Witzig T and Gupta M: Translation initiation complex eIF4F is a therapeutic target for dual mTOR kinase inhibitors in non-Hodgkin lymphoma. Oncotarget 6: 9488-9501, 2015.

15. Fan S, Li Y, Yue P, Khuri FR and Sun SY: The eIF4E/eIF4G interaction inhibitor 4EGI-1 augments TRAIL-mediated apoptosis through c-FLIP Down-regulation and DR5 induction independent of inhibition of cap-dependent protein translation. Neoplasia 12: 346-356, 2010.

16. She QB, Halilovic E, Ye Q, Zhen W, Shirasawa S, Sasazuki T, Solit DB and Rosen N: 4E-BP1 is a key effector of the oncogenic activation of the AKT and ERK signaling pathways that integrates their function in tumors. Cancer Cell 18: 39-51, 2010.

17. Boussemart L, Malka-Mahieu H, Girault I, Allard D, Hemmingsson O, Tomasic G, Thomas M, Basmadjian C, Ribeiro N, Thuaud F, et al: eIF4F is a nexus of resistance to anti-BRAF and anti-MEK cancer therapies. Nature 513: 105-109, 2014.
18. Livak KJ and Schmittgen TD: Analysis of relative gene expression data using real-time quantitative PCR and the 2(-Delta Delta C(T)) method. Methods 25: 402-408, 2001

19. Karthikeyan S and Hoti SL: Development of fourth generation ABC inhibitors from natural products: A novel approach to overcome cancer multidrug resistance. Anticancer Agents Med Chem 15: 605-615, 2015.

20. Bartel DP: MicroRNAs: Genomics, biogenesis, mechanism, and function. Cell 116: 281-297, 2004.

21. Ween MP, Armstrong MA, Oehler MK and Ricciardelli C: The role of $\mathrm{ABC}$ transporters in ovarian cancer progression and chemoresistance. Crit Rev Oncol Hematol 96: 220-256, 2015.

22. Szakács G, Paterson JK, Ludwig JA, Booth-Genthe C and Gottesman MM: Targeting multidrug resistance in cancer. Nat Rev Drug Discov 5: 219-234, 2006.

23. Baguley BC: Multiple drug resistance mechanisms in cancer Mol Biotechnol 46: 308-316, 2010.

24. Liu P, Cheng H, Roberts TM and Zhao JJ: Targeting the phosphoinositide 3-kinase pathway in cancer. Nat Rev Drug Discov 8: 627-644, 2009.

25. Mazan-Mamczarz K, Zhao XF, Dai B, Steinhardt JJ, Peroutka RJ, Berk KL, Landon AL, Sadowska M, Zhang Y, Lehrmann E, et al: Down-regulation of eIF4GII by miR-520c-3p represses diffuse large B cell lymphoma development. PLoS Genet 10: e1004105, 2014.

26. Ho BC, Yu SL, Chen JJ, Chang SY, Yan BS, Hong QS, Singh S, Kao CL, Chen HY, Su KY, et al: Enterovirus-induced miR-141 contributes to shutoff of host protein translation by targeting the translation initiation factor eIF4E. Cell Host Microbe 9: 58-69, 2011.

27. Shi W, Gerster K, Alajez NM, Tsang J, Waldron L, Pintilie M, Hui AB, Sykes J, P'ng C, Miller N, et al: MicroRNA-301 mediates proliferation and invasion in human breast cancer. Cancer Res 71: 2926-2937, 2011.

28. Ma J, Wang T, Guo R, Yang X, Yin J, Yu J, Xiang Q, Pan X, Tang $\mathrm{H}$ and Lei $\mathrm{X}$ : Involvement of miR-133a and miR-326 in ADM resistance of HepG2 through modulating expression of ABCC1. J Drug Target 23: 519-524, 2015.

29. Li H, Zhao J, Zhang JW, Huang QY, Huang JZ, Chi LS, Tang HJ, Liu GQ, Zhu DJ and Ma WM: MicroRNA-217, down-regulated in clear cell renal cell carcinoma and associated with lower survival, suppresses cell proliferation and migration. Neoplasma 60: 511-515, 2013.

30. Tang H, Zhang P, Xiang Q, Yin J, Yu J, Yang X and Lei X: Let-7 g microRNA sensitizes fluorouracil-resistant human hepatoma cells. Pharmazie 69: 287-292, 2014.

31. Borel F, Han R, Visser A, Petry H, van Deventer SJ, Jansen PL and Konstantinova P; Réseau Centre de Ressources Biologiques Foie (French Liver Biobanks Network), France: Adenosine triphosphate-binding cassette transporter genes up-regulation in untreated hepatocellular carcinoma is mediated by cellular microRNAs. Hepatology 55: 821-832, 2012.

32. Xu Y, Xia F, Ma L, Shan J, Shen J, Yang Z, Liu J, Cui Y, Bian X, Bie P and Qian C: MicroRNA-122 sensitizes HCC cancer cells to adriamycin and vincristine through modulating expression of MDR and inducing cell cycle arrest. Cancer Lett 310: 160-169, 2011. 